\title{
Laser Coherence Meter Based on Nanostructured Liquid Crystals
}

\author{
A. Anczykowska, S. Bartkiewicz, and J. Mysliwiec \\ Wroclaw University of Technology, Institute of Physical and Theoretical Chemistry, Wybrzeze Wyspianskiego 27, \\ 50-370 Wroclaw, Poland \\ Correspondence should be addressed to A. Anczykowska; agata.anczykowska@pwr.wroc.pl
}

Received 29 April 2013; Revised 19 July 2013; Accepted 23 July 2013

Academic Editor: Gang-Ding Peng

Copyright ( $2013 \mathrm{~A}$. Anczykowska et al. This is an open access article distributed under the Creative Commons Attribution License, which permits unrestricted use, distribution, and reproduction in any medium, provided the original work is properly cited.

\begin{abstract}
We present the method for coherence length measurement using coherence meter based on hybrid liquid crystal structures doped with gold nanoparticles. The results indicate that the method is able to determine the coherence length of coherent light sources with precision of $0.01 \mathrm{~m}$ at wavelength range from 200 to $800 \mathrm{~nm}$ for wide range of initial beam powers starting from $1 \mathrm{~mW}$. Given the increasing use of laser technology in industry, military, or medicine, our research may open up a possible route for the development of improved techniques of coherent diagnostic light sources.
\end{abstract}

\section{Introduction}

The coherence length of the laser light is the propagation distance from the emission source to the point in which electromagnetic wave maintains a specified degree of coherence. It is proportional to the output power divided by the square of the cavity round-trip time according to the Schawlow-Townes formula, but this limit is rarely achieved under the influence of various noise sources [1]. The coherence is also influenced by drift and environmental factors such as temperature drifts, humidity, or air pressure [2]. Since the commercial solutions for the measurement of the coherence length are not available, the vast majority of such measurements are made by Michelson interferometer [3]. The main drawbacks of this solution are the time-consuming preparations of the measurement system followed by the necessity of complicated manual modifications and complex calculations. Consequently, we still need faster and more efficient measurement technology for the coherence length of laser light.

Since all the remarkable properties of laser light depend on the coherence, determining its length is essential especially when the coherence of the emitted light is specifically large or small. Lasers with large temporal and spatial coherence have found numerous applications, including interferometry, holography, and construction of certain types of optical sensors as well as, coherent beam combining technology, which is used in sectors such as metallurgy, medicine, military, and communication [4-7]. On the other hand, low temporal coherence combined with high spatial coherence is the key parameter of certain optical research techniques such as optical coherent tomography used for imaging of the retina and optic nerve structures $[8,9]$. Presented coherence meter can cover all of the above examples as well as provide the answer to the question whether the tested laser maintains its optical properties over time.

\section{Experiment}

The coherence meter presented in Figure 1(a) is based on the typical degenerated two-wave mixing set-up working principle. The light emitted by the tested laser is split on the beamsplitter into two equal beams $I_{1}$ and $I_{2}$. The optical path of one of the beams $\left(I_{1}\right)$ is extended with delay system comprising second beamsplitter and sliding mirror, which results in the specified phase shift. Beams intensity difference and phase shifts resulting from experimental set-up geometry are compensated with filter and phase compensator. Optical path difference resulting from uneven set-up geometry is given as $l_{\min }=2 x_{J} \cdot\left(n_{J}-1\right)+2 x_{K}$, where $x_{J}$ is the beamsplitter thickness, $n_{J}$ is beamsplitter refractive index, and $x_{K}$ is minimum distance between sliding mirror and the beamsplitter. Phase compensator $C$ is adjusted to cover this path difference resulting in simultaneous arrival of beams to the LC modulator when $\Delta x=0$. Consequently the 


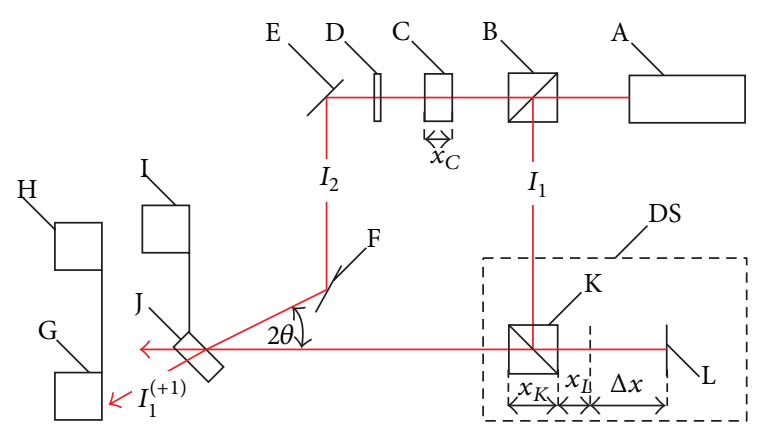

(a)

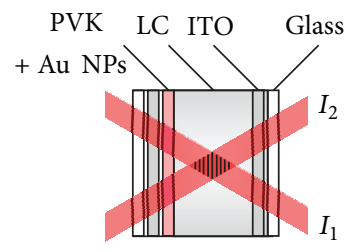

(b)

Figure 1: Scheme of the coherence meter prototype (a) and spatial light modulator (b). A: tested laser, B, K: beamsplitter, C: phase compensator, D: filter, E, F: mirror, G: photodiode, H: voltmeter, I: DC voltage supplier, J: spatial light modulator, L: sliding mirror, and DS: delay system. $\mathrm{DC}=20 \mathrm{~V}, 2 \theta=0.17 \mathrm{rad}$.

compensatory path difference is given as $l_{\min }^{\prime}=\left(n_{C}-1\right) x_{C}$, where $n_{C}$ is the phase compensator refractive index and $x_{C}$ is the thickness. Subsequently the beams recombine in the dynamic holographic material powered by the DC, where they interfere. The photodiode measures the light intensity in the plus first diffraction order: then the data is transferred and displayed on the voltmeter [10]. The experimental set-up geometry was chosen based on the previous research determining the configuration ensuring the highest diffraction efficiency. The angle between the beams was set to $0.17 \mathrm{rad}$, the voltage was set to $20 \mathrm{~V}$, and the sample twist angle was set to $0 \mathrm{rad}$ to avoid energy transfer between the beams [11]. The maximum sliding mirror range $\Delta x$ was fixed at $1.25 \mathrm{~m}$ according to available space restrictions.

The key component of presented coherence meter is the spatial light modulator used to strengthen the interference pattern created by two interfering beams, record it, and as a result diffract the incident light, all of which take place within 100-300 ms required for the signal stabilization. The modulator comprises liquid crystal, photoconducting layer, and two glass plates covered with indium tin oxide (ITO). The most efficient configuration of modulator parameters was chosen based on previous studies on the influence of metal nanoparticles and quantum dots on the properties of liquid crystal structures [11]. The configuration ensuring the highest diffraction efficiency combined with the shortest response time was achieved for the $5 \mu \mathrm{m}$ thick LC structure with photoconducting layer of polyvinylcarbazole (PVK) doped with $\mathrm{Au}$ nanoparticles (Au NPs) at mass ratio of $1: 1000$. Its schematic structure is shown in Figure 1(b).

Gold nanoparticles used in experiments were Nsol nanorods (Nanopartz), with maximum absorption at $\lambda=$ $650 \mathrm{~nm}$ and axial size of $25 \mathrm{~nm}$. The NPs were equipped with polymeric layer providing long-suspension times in organic solvents. Nematic LC mixture 1658 (AWAT Poland) was characterized at room temperature by birefringence $\Delta n=$ 0.380 at $633 \mathrm{~nm}$, positive dielectric anisotropy $\Delta \varepsilon=15.6$, crystallization temperature $T_{c}=-20^{\circ} \mathrm{C}$, and isotropization temperature $T_{i}=114^{\circ} \mathrm{C}[12]$.

\section{Results}

Presented coherence meter measures the intensity of one of the interfering beams in the plus first diffraction order $\left(I_{1}^{(+1)}\right)$, which is later converted to the diffraction efficiency of the system defined as $\eta=I_{1}^{(+1)} / I_{1} \cdot 100 \%$, where $I_{1}$ is the initial intensity of one of the interfering beams. The interference can be observed as long as the beams are coherent; in other words, the time delay is such that $\Delta t \sim 1 / \Delta v$, where $\Delta v$ is bandwidth of the light. Consequently the coherence length would be given as $\Delta s=2 \Delta x=c \Delta t$ [13]. When the difference between the optical paths of the beams $\Delta s$ reaches certain point understood as the coherence length, diffraction efficiency of the system would rapidly drop. Since the coherence of the laser is proportional to the diffraction efficiency of the system/interference pattern intensity, the objective of the experiments was to find the optical path length difference of a self-interfering laser beam, which reduces the diffraction efficiency/interference pattern intensity to $1 / e^{2}$ of its maximum value [14]. The measurements were taken for one laser and two laser diodes. Each light source specification can be found in Table 1.

The results (Figure 2) show that the diffraction efficiency obtained for different coherent light sources maintains its value within certain range of the path difference between the beams, then starts to decrease rapidly, and finally reaches 0 .

Calculated coherence length of the diode I is $1.00 \mathrm{~m}$, whilst diode II has the coherence length of $0.14 \mathrm{~m}$. The coherence length of Nd:YAG laser was impossible to be determined at this stage of research because of method limitationsdiffraction efficiency within $2.5 \mathrm{~m}$ distance remained constant at its value. What is interesting is that registered efficiency is relatively high even for laser diodes with very low power, which ensures high sensitivity of the method. Consequently, it can be applied to the coherence measurements of low-energy light sources as well as high-energy light sources, in which case the power can be easily reduced to avoid modulator overheating and eventual electrical breakdowns. 
TABLE 1: Technical specification of tested light sources.

\begin{tabular}{lcccc}
\hline Name & Wavelength $(\mathrm{nm})$ & Power $(\mathrm{mW})$ & Type & Coherence length $(\mathrm{m})$ \\
\hline Laser & 532 & 150 & Nd:YAG & $>2.50$ \\
diode I & 650 & 10 & Semiconductor diode & 1.00 \\
diode II & 635 & 5 & Semiconductor diode & 0.14 \\
\hline
\end{tabular}

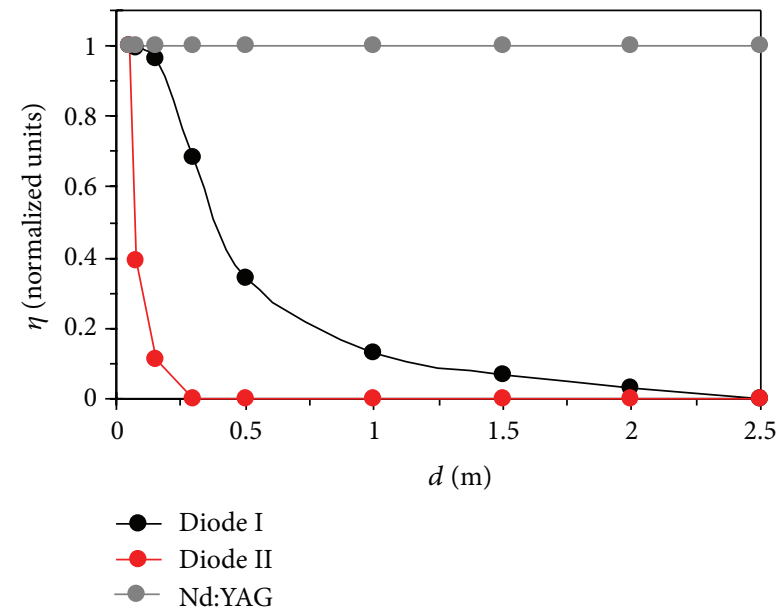

FIGURE 2: Normalized diffraction efficiency dependence on the optical paths difference of a self-interfering laser beam for tested coherent light sources.

Currently, presented method is limited to coherent light sources whose coherence length is shorter than the double of sliding mirror range $2 \Delta x=2.5 \mathrm{~m}$, which is expanded by delay system modifications, that is, by using optical fiber system. Second limitation is the accuracy of measurements currently fixed at $0.01 \mathrm{~m}$, but introducing phase modulator can allow the measurement of coherence lengths at nanometer scale. Final limitation of presented coherence meter comes with the initial usage of glass plates which absorb most of the UV spectra, but this can be resolved by using quartz glasses for SLM preparation. Infrared spectrum is also available by introducing nanoparticles with maximum absorption within IR range. As of now, presented device can be applied to measure the coherence of light sources at any wavelength within visible and near-UV spectrum.

\section{Conclusion}

In this paper, we presented initial results of coherence length measurement obtained with proposed coherence meter based on hybrid liquid crystal structures doped with gold nanoparticles. Presented method can be applied to coherence measurement of light sources within wide energy range starting at less than $1 \mathrm{~mW}$ with no upper limitations. It can also be applied to measure the coherence of light sources at any wavelength within visible and near-UV spectrum.

\section{Acknowledgments}

The authors wish to thank Wroclaw University of Technology and the Polish National Science Center for financial support of the project awarded on the basis of the Decision nos. DEC-2011/03/B/ST5/01021, 2011/01/B/ST8/03317, and 2011/01/ B/ST5/00773.

\section{References}

[1] W. Elsaesser and E. O. Goebel, "Multimode effects in the spectral linewidth of semiconductor lasers," IEEE Journal of Quantum Electronics, vol. 21, no. 6, pp. 687-692, 1985.

[2] S. Roling, B. Siemer, M. Wöstmann et al., "Temporal and spatial coherence properties of free-electron-laser pulses in the extreme ultraviolet regime," Physical Review Special Topics, vol. 14, no. 8, Article ID 080701, 2011.

[3] P. G. Kwiat, W. A. Vareka, C. K. Hong, H. Nathel, and R. Y. Chiao, "Correlated two-photon interference in a dual-beam Michelson interferometer," Physical Review A, vol. 41, no. 5, pp. 2910-2913, 1990.

[4] R. F. Smith, J. Dunn, J. Nilsen et al., "Picosecond x-ray laser interferometry of dense plasmas," Physical Review Letters, vol. 89, no. 6, Article ID 065004, 2002.

[5] Q. Lou, J. Zhou, B. He, and H. Zhao, "Fiber lasers and their coherent beam combination," Optics and Photonics News, vol. 19, no. 5, pp. 46-51, 2008.

[6] W. M. Wang, K. T. V. Grattan, A. W. Palmer, and W. J. O. Boyle, "Self-mixing interference inside a single-mode diode laser for optical sensing applications," Journal of Lightwave Technology, vol. 12, no. 9, pp. 1577-1587, 1994.

[7] S. J. Augst, T. Y. Fan, and A. Sanchez, "Coherent beam combining and phase noise measurements of ytterbium fiber amplifiers," Optics Letters, vol. 29, no. 5, pp. 474-476, 2004.

[8] E. Regara, J. A. Schaara, E. Montb, R. Virmanib, and P. W. Serruys, "Optical coherence tomography," Cardiovascular Radiation Medicine, vol. 4, pp. 198-204, 2003.

[9] A. F. Fercher and C. K. Hitzenberger, "Chapter 4 Optical coherence tomography," Progress in Optics, vol. 44, pp. 215-302, 2002.

[10] S. Bartkiewicz and A. Anczykowska, "Polish patent submission: method for coherence measurements of light sources and coherence meter," MKP Classification: G01J9/00G01J9/02, Submission number: 387382, 2009.

[11] A. Anczykowska, S. Bartkiewicz, M. Nyk, and J. Myśliwiec, "Enhanced photorefractive effect in liquid crystal structures codoped with semiconductor quantum dots and metallic nanoparticles," Applied Physics Letters, vol. 99, no. 19, Article ID 191109, 2011.

[12] A. Anczykowska, S. Bartkiewicz, M. Nyk, and J. Mysliwiec, "Study of semiconductor quantum dots influence on photorefractivity of liquid crystals," Applied Physics Letters, vol. 101, Article ID 101107, 4 pages, 2012.

[13] W. Demtröder, Laser Spectroscopy: Vol. 1: Basic Principles, Springer, Berlin, Germany, 2008.

[14] J. M. Khosrofian and B. A. Garetz, "Measurement of a Gaussian laser beam diameter through the direct inversion of knife-edge data," Applied Optics, vol. 22, no. 1, pp. 3406-3410, 1983. 

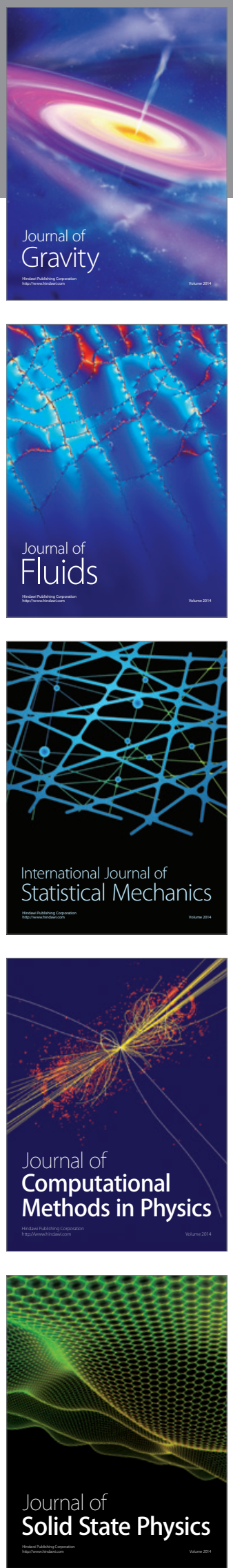

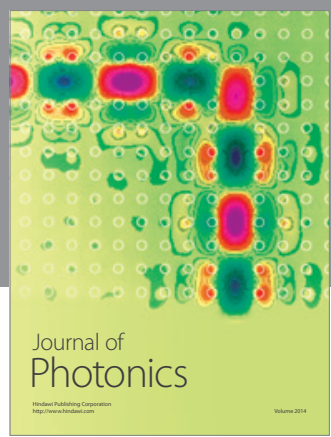

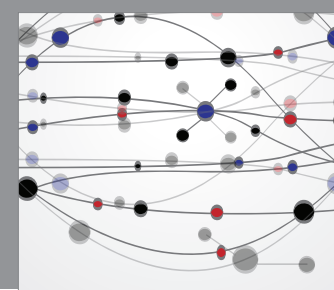

The Scientific World Journal

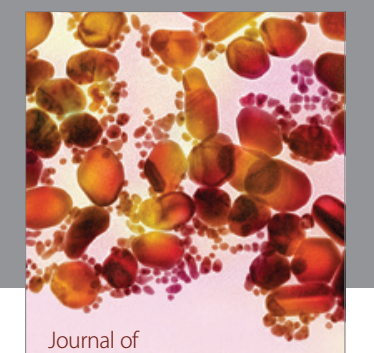

Soft Matter
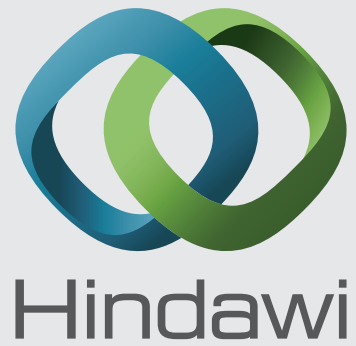

Submit your manuscripts at

http://www.hindawi.com
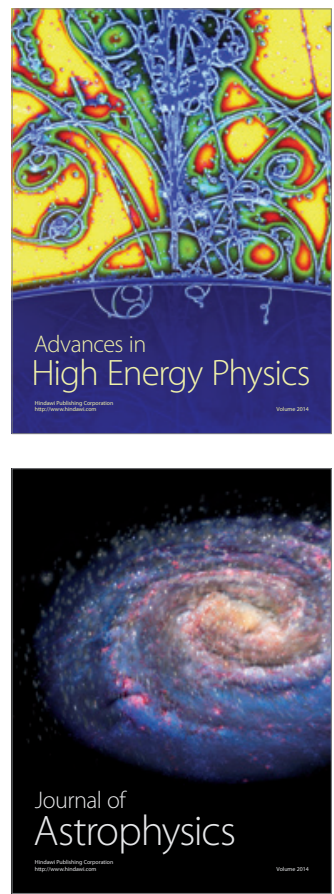
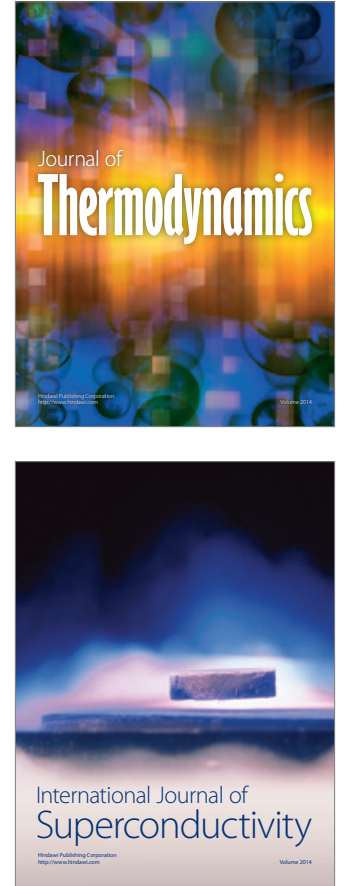
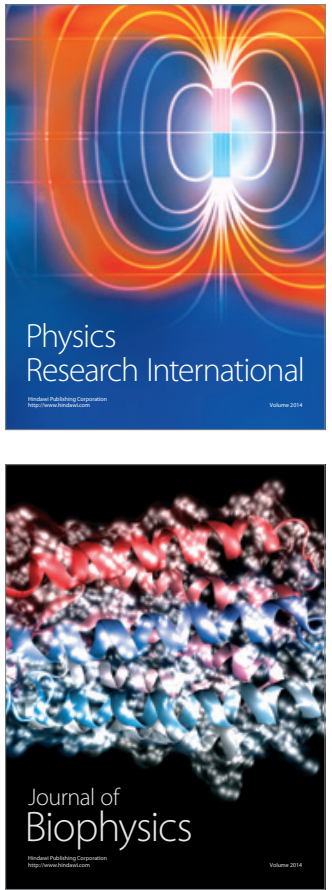
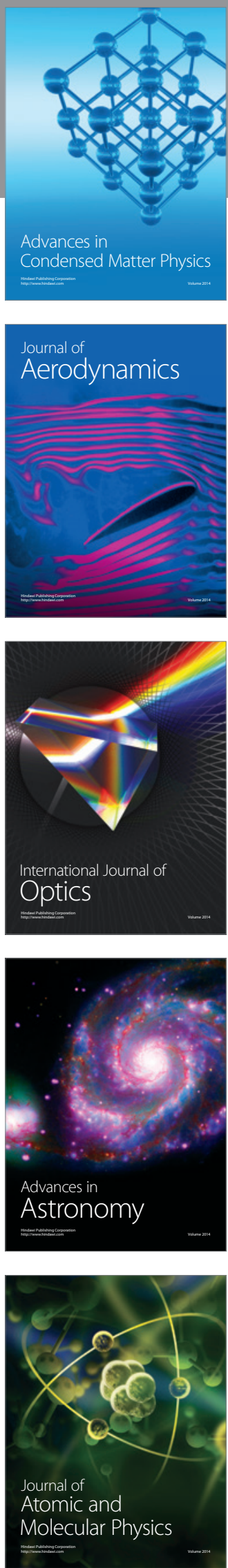